\title{
Multiple-Surrogate Approach to Helicopter Rotor Blade Vibration Reduction
}

\author{
Bryan Glaz* \\ University of Michigan, Ann Arbor, Michigan 48109 \\ Tushar Goelı \\ University of Florida, Gainesville, Florida 32611 \\ Li Liu and Peretz P. Friedmann $\stackrel{\S}{\S}$ \\ University of Michigan, Ann Arbor, Michigan 48109 \\ and \\ Raphael T. HaftkaI \\ University of Florida, Gainesville, Florida 32611
}

DOI: $\underline{10.2514 / 1.40291}$

\begin{abstract}
The advantages of using multiple surrogates for approximation and reduction of helicopter vibration are studied. Multiple approximation methods, including a weighted-average approach, are considered so that pitfalls associated with only using a single best surrogate for the rotor blade vibration-reduction problem are avoided. A vibration objective function corresponding to a flight condition in which blade-vortex interaction causes high levels of vibration is considered. The design variables consist of cross-sectional dimensions of the structural member of the blade and nonstructural masses. The optimized designs are compared with a baseline design resembling a Messerschmitt-Bölkow-Blohm BO-105 blade. The results indicate that at relatively little additional cost compared with optimizing a single surrogate, multiple surrogates can be used to locate various reduced-vibration designs that would be overlooked if only a single approximation method was employed, and the most accurate surrogate may not lead to the best design.
\end{abstract}

$C_{d f}$
$C_{d 0}$
$C_{W}$
$c$
$\mathbf{D}$
$d_{1}, d_{2}$
$E$
$E_{\text {avg }}$
$E_{i}$
$F_{4 X}, F_{4 Y}, F_{4 Z}$
$\hat{F}_{4 X}, \hat{F}_{4 Y}, \hat{F}_{4 Z}$

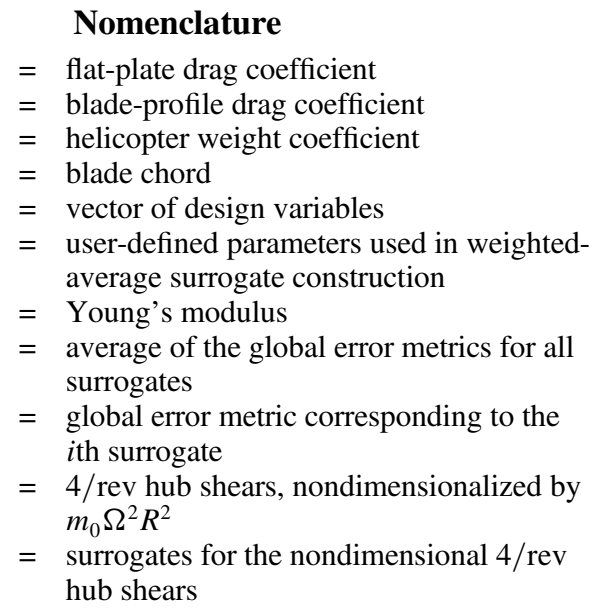

Presented as Paper 1898 at the 48th AIAA/ASME/ASCHE/AHS/ASC Structures, Structural Dynamics and Materials Conference, Honolulu, HI, 23-26 April 2007; received 6 August 2008; accepted for publication 20 August 2008. Copyright $\odot 2008$ by B. Glaz, T. Goel, L. Liu, P. P. Friedmann, and R. T. Haftka. Published by the American Institute of Aeronautics and Astronautics, Inc., with permission. Copies of this paper may be made for personal or internal use, on condition that the copier pay the $\$ 10.00$ per-copy fee to the Copyright Clearance Center, Inc., 222 Rosewood Drive, Danvers, MA 01923; include the code 0001-1452/09 \$10.00 in correspondence with the CCC.

*Postdoctoral Researcher, Department of Aerospace Engineering. Member AIAA.

${ }^{\dagger}$ Department of Mechanical and Aerospace Engineering; currently Scientist, Livermore Software Technology Corporation. Member AIAA.

${ }^{\ddagger}$ Postdoctoral Researcher, Department of Aerospace Engineering. Member AIAA.

${ }^{\S}$ François-Xavier Bagnoud Professor, Department of Aerospace Engineering. Fellow AIAA.

"Distinguished Professor, Department of Mechanical and Aerospace Engineering. Fellow AIAA.
$\mathrm{GMSE}_{i}$

$f(\mathbf{x})$
$g(\mathbf{D})$
$h$
$J$
$\hat{J}$
$J_{P}$
$M_{4 X}, M_{4 Y}, M_{4 Z}$
$\hat{M}_{4 X}, \hat{M}_{4 Y}, \hat{M}_{4 Z}$
$m_{\mathrm{ns}}$
$m_{0}$
$N_{b}$
$N_{c}$
$N_{\mathrm{dv}}$
$N_{\mathrm{RBF}}$

$N_{\text {sm }}$

$N_{\text {sp }}$

$N_{\mathrm{tp}}$

$t_{1}, t_{2}, t_{3}$

$w_{i}$

$w_{\text {poly }}, w_{\text {krg }}, w_{\text {RBNN }}$

$\mathbf{x}^{(i)}$

$x_{1}, x_{2}$

$X_{F A}, Z_{F A}$

$X_{F C}, Z_{F C}$
$=$ generalized mean square error corresponding to the $i$ th surrogate

$=$ assumed polynomials that account for the global behavior in kriging

$=$ constraints

$=$ height of the blade cross section

$=$ objective function

$=$ surrogate objective function

$=$ mass polar moment of inertia of the rotor

$=4 /$ rev hub moments, nondimensionalized by $m_{0} \Omega^{2} R^{3}$

$=$ surrogates for the nondimensional $4 / \mathrm{rev}$ hub moments

$=$ nonstructural mass located at the elastic axis

$=$ baseline mass per unit length

$=$ number of rotor blades

$=$ number of behavior constraints

$=$ number of design variables

$=$ number of radial basis functions associated with radial basis neural networks

$=$ number of surrogate models

$=$ number of sample points

$=$ number of test points

$=$ blade radius

$=$ thicknesses of the blade cross section

$=$ weight coefficient corresponding to the $i$ th surrogate

$=$ weight coefficients corresponding to each surrogate

$=i$ th sample point

$=$ cross-sectional dimensions

$=$ longitudinal and vertical offsets between rotor hub and helicopter aerodynamic center

$=$ longitudinal and vertical offsets between rotor hub and helicopter center of gravity 


\begin{tabular}{|c|c|}
\hline $\bar{y}$ & $=$ factor used to normalize errors \\
\hline$y(\mathbf{x})$ & $=$ unknown function to be approximated \\
\hline$\hat{y}(\mathbf{x})$ & $=$ approximation of $y(\mathbf{x})$ \\
\hline$y^{(i)}$ & $=$ output response at $\mathbf{x}^{(i)}$ \\
\hline$Z(\mathbf{x})$ & $\begin{array}{l}=\text { realization of a stochastic process in } \\
\text { kriging }\end{array}$ \\
\hline$\alpha_{d}$ & $=$ flight descent angle \\
\hline$\alpha_{i}$ & $\begin{array}{l}=\text { weight associated with the } i \text { th radial basis } \\
\text { function in radial basis neural networks }\end{array}$ \\
\hline$\beta_{p}$ & $=$ blade precone angle \\
\hline$\beta_{0}, \beta_{i}, \beta_{i j}$ & $\begin{array}{l}=\text { fitting coefficients in polynomial } \\
\text { regression }\end{array}$ \\
\hline$\varepsilon$ & $\begin{aligned} &= \text { absolute percent error of surrogate } \\
& \text { predictions }\end{aligned}$ \\
\hline$\epsilon_{\mathrm{pr}}$ & $\begin{array}{l}=\text { approximation error in polynomial } \\
\text { regression }\end{array}$ \\
\hline$\zeta_{k}, \omega_{k}$ & $=$ real and imaginary parts of $\lambda_{k}$ \\
\hline$\theta_{\mathrm{pt}}$ & $=$ blade built-in pretwist angle \\
\hline$\lambda_{k}$ & $=$ hover-stability eigenvalue for $k$ th mode \\
\hline$\mu$ & $=$ advance ratio \\
\hline$v$ & $=$ Poisson's ratio \\
\hline$\rho_{\text {filler }}$ & $\begin{array}{l}=\text { material density for nonstructural filler } \\
\text { mass }\end{array}$ \\
\hline$\rho_{\text {struct }}$ & $\begin{array}{l}=\text { material density for the structural member } \\
\text { of the blade }\end{array}$ \\
\hline$\sigma$ & $=$ rotor solidity \\
\hline$\sigma_{\text {allowable }}$ & $=$ allowable blade stress \\
\hline$\sigma_{x x}, \sigma_{x \eta}, \sigma_{x \zeta}$ & $=$ blade stresses \\
\hline$\sigma_{Y}$ & $=$ yield stresses \\
\hline$\tau$ & $\begin{array}{l}=\text { parameter that controls the radius of } \\
\text { influence for each neuron in radial basis } \\
\text { neural networks }\end{array}$ \\
\hline$\phi_{\mathrm{RBNN}}$ & $\begin{array}{l}=\text { radial basis functions used in radial basis } \\
\text { neural networks }\end{array}$ \\
\hline$\Omega$ & $=$ rotor angular speed \\
\hline$\omega_{F 1}, \omega_{L 1}, \omega_{T 1}$ & $\begin{array}{l}=\text { fundamental rotating-flap, lead-lag, and } \\
\text { torsional frequencies per revolution }\end{array}$ \\
\hline$\omega_{L}, \omega_{U}$ & $\begin{array}{l}=\text { lower and upper bounds for frequency } \\
\text { constraints per revolution }\end{array}$ \\
\hline
\end{tabular}

\section{Introduction}

$\mathbf{V}$ IBRATION is one of the most critical concerns in the design of modern rotorcraft. Stricter demands for enhanced performance, comfort, and customer acceptance require designs with reduced vibration levels. In helicopters, the dominant source of vibrations is the rotor, which transfers vibrations to the rotor hub and fuselage at harmonics that are predominantly integer multiples of $N_{b} / \mathrm{rev}$, where $N_{b}$ is the number of blades.

During the last 25 years, two principal approaches to vibration reduction have emerged. The first approach is passive and uses structural/multidisciplinary optimization for reducing vibrations [13]. The second approach uses active control methods [4]. The passive approach is used by blade designers to improve the vibration characteristics of the rotor. On the other hand, the active approach is still considered to be a research topic that is slowly approaching implementation. This study is aimed at the passive approach, in which the vibration-reduction problem is formulated as a mathematical optimization problem subject to appropriate constraints. The objective function consists of a suitable combination of the $N_{b} /$ rev hub shears and moments that are computed from an aeroelastic response code; constraints are specified on blade stability margins, frequencies, blade geometry, autorotational properties, and blade stresses. The design variables can be dimensions of the blade cross section, mass and stiffness distributions along the span, or geometrical parameters that define advance geometry tips. Typical levels of vibration reduction achieved with passive approaches have been in the range of $30-60 \%$ relative to a baseline design.

Because of the complex rotary-wing aerodynamic environment, the aeroelastic response simulations needed for vibratory-load calculations are computationally expensive. Therefore, numerous evaluations of the vibration objective function are costly. Consequently, direct combination of the objective function generated by an aeroelastic response simulation with traditional optimization algorithms is computationally very expensive. Moreover, traditional optimization search algorithms can converge to local optima, which are known to occur in this class of problems.

To overcome these obstacles, global approximation concepts have been used [5-7]. A typical approach when using approximation, or surrogate methods, is to assess the performance of various surrogate models and then select the one that appears to be most accurate. This method was recently applied to the helicopter vibration-reduction problem in [8], in which second-order polynomial response surfaces, radial basis function interpolation, and kriging were considered for construction of the vibratory-hub-load surrogates. The study was based on a comprehensive helicopter simulation code that uses advanced modeling techniques such as free-wake modeling. Seventeen design variables were used to characterize the blade's spanwise mass and stiffness distributions, including blade crosssectional dimensions and nonstructural masses. For the cases considered, the results showed that the kriging surrogate was generally the most accurate global approximation method and led to the best designs. However, no single approximation method distinguished itself as the best for all cases.

Most research dealing with surrogate modeling has been concerned with selecting a single approximation method among different approaches. However, the choice of the best surrogate model is determined by a number of factors, and once selected, the choice of the best approximation method is seldom reviewed. These factors include the number of points used to construct the surrogate model (sampling density), the scheme used to select points (design of experiments), and parameters/nature of the surrogate model. Different surrogate models have been shown to perform well under different conditions and for different objectives. For instance, the most accurate approximation method may not necessarily lead to the best design. Thus, a single approximation method has not distinguished itself as the most suitable for engineering applications.

As an alternative to seeking the best approximation method, there has been recent work on the collaborative use of an ensemble of surrogates [9-11]. Use of multiple surrogates is motivated by our inability to find a unique solution to the nonlinear inverse problem of identifying the model from a limited set of data [5] and essentially serves as an approach to account for the uncertainty in the choice of the appropriate approximation methods. Typically, the cost of obtaining fitting data required for developing surrogates is high. So it is desirable to extract as much information as possible from the data. Although selecting the fitting parameters may require substantial effort for certain approximation methods, surrogates can often be constructed without significant expense, compared with the cost of acquiring data. Therefore, the use of an ensemble of surrogates can prove to be a relatively inexpensive method of distilling correct trends from the data while protecting against poor surrogate models. In addition, using multiple surrogates decreases the likelihood of overlooking promising regions in the design space, because different approximation methods may favor different designs.

The objectives of this paper are to demonstrate the advantages of using multiple surrogates, which results in little additional cost compared with using only one approximation method, along with the applicability of such methods to the helicopter vibration-reduction problem. Furthermore, pitfalls associated with using a single approximation method are exemplified using the rotor blade vibration-reduction problem.

\section{Overview of the Aeroelastic Response and Stability Analysis}

The simulation code used in this study is based on a comprehensive aeroelastic analysis code [12-14]. The aeroelastic response analysis can represent the behavior of hingeless rotor blades, as shown in Fig. 1, with actively controlled flaps. The key ingredients of the aeroelastic response analysis are 1) the structural dynamic model, 2) the unsteady aerodynamic model, and 3) a 


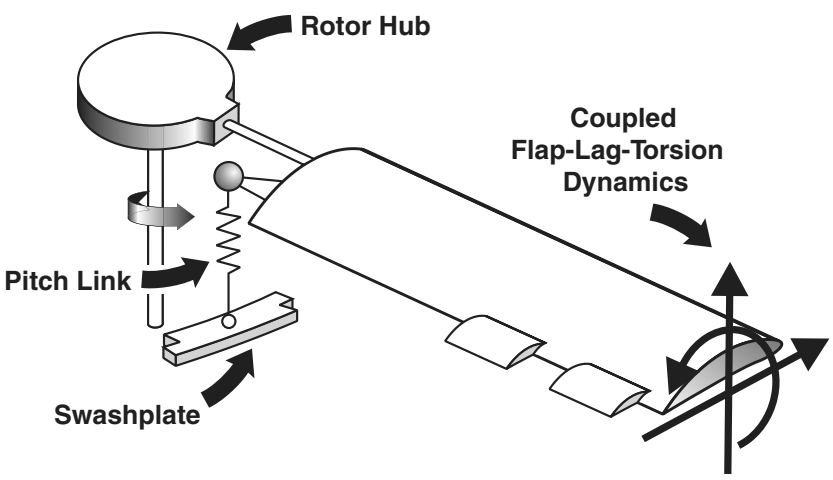

Fig. 1 Helicopter rotor blade with trailing-edge flaps.

coupled trim/aeroelastic response procedure that is required for the computation of the steady-state blade response. The aeroelastic response analysis and overviews of the blade stress calculations and aeroelastic stability in hover analysis are described next.

\section{A. Structural Dynamic Model}

The structural dynamic model is based on an analysis developed by Yuan and Friedmann $[12,15]$ that is capable of modeling composite blades with transverse shear deformations, crosssectional warping, and swept tips. This study is limited to the behavior of isotropic blades with spanwise-varying properties. The equations of motion are formulated using a finite element discretization of Hamilton's principle, with the assumption that the blade undergoes moderate deflections. The beam type finite elements used for the discretization have 23 nodal degrees of freedom. Normal modes are used to reduce the number of structural degrees of freedom. In this study, 8 modes are used: the first 3 flap modes, first 2 lead-lag modes, first 2 torsional modes, and first axial mode.

\section{B. Aerodynamic Model}

The attached-flow blade-section aerodynamics are calculated using a rational function approach (RFA) [13]. The RFA approach is a two-dimensional unsteady time-domain theory that accounts for compressibility as well as variations in the oncoming flow velocity. This two-dimensional aerodynamic model is linked to an enhanced free-wake model that provides a nonuniform inflow distribution at closely spaced azimuthal steps $[16,17]$. Although the simulation code can also account for dynamic stall at high advance ratios, dynamic stall was not considered in this paper because the vibration levels being approximated are those due to blade-vortex interaction (BVI), which occurs at low advance ratios.

\section{Coupled Trim/Aeroelastic Response}

The combined structural and aerodynamic equations form a system of coupled ordinary differential equations that are cast into first-order state-variable form and integrated in the time domain using the Adams-Bashforth predictor-corrector algorithm [18]. A propulsive-trim procedure, in which six equilibrium equations (three forces and three moments) are enforced, is used in this study. The trim equations are solved in a coupled manner with the aeroelastic equations of motion. The vibratory-hub shears and moments are found by integrating the distributed inertial and aerodynamic loads over the entire blade span in the rotating frame, then transforming these loads to the hub-fixed nonrotating system and summing the contributions from each blade [15]. In the process, cancellation of various terms occurs and the primary components of the hub shears and moments have a frequency of $N_{b} / \mathrm{rev}$, which is known as the blade passage frequency.

\section{Blade Stresses}

The procedure for calculating stresses is as follows:
1) For a given azimuth angle, the displacements at any spanwise location are calculated by the aeroelastic response code.

2) The displacements are then substituted into the nonlinear straindisplacement relations [15], giving the strains at any spanwise location.

3) Stresses are calculated from the stress-strain relations.

This calculation gives the blade stresses at any spanwise location and at any azimuth angle.

\section{E. Aeroelastic Stability in Hover}

The process for determining the hover stability of the blade is based on the method used in [15] and is described next:

1) The nonlinear static equilibrium solution of the blade is found for a given pitch setting and uniform inflow by solving a set of nonlinear algebraic equations. Note that uniform inflow is used only in the hover-stability calculation. The forward-flight analysis employs a free-wake model for inflow calculation.

2) The governing system of ordinary differential equations is linearized about the static equilibrium solution by writing perturbation equations and neglecting second-order-and-higher terms in the perturbed quantities. The linearized equations are rewritten in first-order state-variable form.

3) The real parts of the eigenvalues of the first-order state-variable matrix, $\lambda_{k}=\zeta_{k}+i \omega_{k}$, determine the stability of the system. If $\zeta_{k} \leq 0$ for all $k$, the system is stable.

For this study, the linearization process from [15] is modified to account for the aerodynamic states introduced by the RFA model [].

\section{Formulation of the Blade Optimization Problem}

The formulation of the blade optimization problem in forward flight consists of several ingredients: the objective function, design variables, and constraints. The mathematical formulation of the optimization is stated as follows. Find the vector of design variables $\mathbf{D}$ that minimizes the objective function [i.e., $J(\mathbf{D}) \rightarrow$ min], where the objective function consists of a combination of the $N_{b} /$ rev oscillatory-hub shears and moments. For a four-bladed rotor, the objective function is given by

$$
\begin{aligned}
J= & K_{S} \sqrt{\left(F_{4 X}\right)^{2}+\left(F_{4 Y}\right)^{2}+\left(F_{4 Z}\right)^{2}} \\
& +K_{M} \sqrt{\left(M_{4 X}\right)^{2}+\left(M_{4 Y}\right)^{2}+\left(M_{4 Z}\right)^{2}}
\end{aligned}
$$

where $K_{S}$ and $K_{M}$ are appropriately selected weighting factors.

The vector of design variables $\mathbf{D}$ consists of the thicknesses $t_{1}, t_{2}$, and $t_{3}$ and the nonstructural mass $m_{\mathrm{ns}}$ located at the shear center, which are specified at several spanwise locations and shown in Fig. 2. The three thickness design variables were defined at the $0,25,50,7 \overline{5}$, and $100 \%$ stations, and the nonstructural mass design variable was defined at the 68 and $100 \%$ blade stations, resulting in a total of 17 design variables. These two blade stations were chosen for the nonstructural mass because previous studies have shown that nonstructural masses are most effective for vibration reduction when they are distributed over the outboard one-third of the blade $[\underline{19}, \underline{20}]$. The cross-sectional variables were assumed to vary linearly between stations. The nonstructural mass at the elastic axis inboard of the $68 \%$ station was set to zero. The design variables have side constraints to prevent them from reaching impractical values; these are stated as

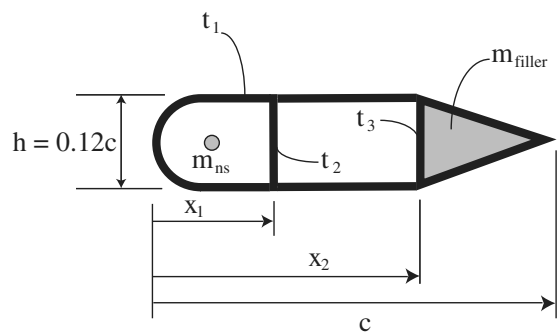

Fig. 2 Simplified model of the blade structural member. 


$$
\mathbf{D}_{j}^{(L)} \leq \mathbf{D} \leq \mathbf{D}_{j}^{(U)}, \quad j=1,2, \ldots, N_{\mathrm{dv}}
$$

In addition, four types of behavior constraints, given by

$$
g_{i}(\mathbf{D}) \leq 0, \quad i=1,2, \ldots, N_{c}
$$

are placed on the blade design. The first behavior constraints are frequency-placement constraints, which are prescribed upper and lower bounds on the fundamental flap, lag, and torsional frequencies of the blade. The frequency-placement constraints on the fundamental flap frequency are written as

$$
g_{\text {flap }}(\mathbf{D})=\frac{\omega_{F 1}}{\omega_{U}}-1 \leq 0
$$

and

$$
g_{\text {flap }}(\mathbf{D})=1-\frac{\omega_{F 1}}{\omega_{L}} \leq 0
$$

where $\omega_{U}$ and $\omega_{L}$ are the prescribed upper and lower bounds on the fundamental flap frequency. Similar constraints are placed on the lag and torsional frequencies (i.e., $g_{\text {lag }}$ and $g_{\text {torsion }}$ ). In addition, all blade frequencies must differ from integer multiples of the angular velocity ( $1 / \mathrm{rev}, 2 / \mathrm{rev}, 3 / \mathrm{rev}$, etc.) to avoid undesirable resonances.

Another behavior constraint is an autorotational constraint, which ensures that mass redistributions produced during the optimization do not degrade the autorotational properties of the rotor. Although there are several indices that can be used to represent the autorotational properties of the blade, the one used in this study is to require that the mass polar moment of inertia of the rotor be at least $90 \%$ of its baseline value. Mathematically, this is expressed as

$$
g_{a}(\mathbf{D})=1-\frac{J_{P}}{0.9 J_{P 0}} \leq 0
$$

where $J_{P}$ is the mass polar moment of inertia of the rotor when it is spinning about the shaft, and $J_{P 0}$ is the baseline value.

The third behavior constraints are aeroelastic stability-margin constraints, expressed mathematically as

$$
g_{k}(\mathbf{D})=\zeta_{k}+\left(\zeta_{k}\right)_{\min } \leq 0, \quad k=1,2, \ldots, N_{m}
$$

where $N_{m}$ is the number of normal modes, $\zeta_{k}$ is the real part of the hover eigenvalue for the $k$ th mode, and $\left(\zeta_{k}\right)_{\min }$ is the minimum acceptable damping level for the $k$ th mode. It should be noted that the most critical modes for stability are usually the first and second lag modes.

The final behavior constraint is a stress constraint obtained by substituting the blade stresses into von Mises' criterion, which is expressed mathematically as

$$
\frac{2 \sigma_{x x}^{2}+6\left(\sigma_{x \eta}^{2}+\sigma_{x \zeta}^{2}\right)}{6}-\frac{\sigma_{\text {allowable }}^{2}}{3} \leq 0
$$

where $\sigma_{x x}, \sigma_{x \eta}$, and $\sigma_{x \zeta}$ are the axial and shear stresses, and $\sigma_{\text {allowable }}$ is the material yield stress divided by a factor of safety. At discrete values of the azimuth angle, Eq. ( $\underline{8})$ is evaluated at spanwise locations corresponding to the finite element nodes. The maximum evaluation of Eq. ( $\underline{8}$ ) is used for the constraint and is given as

$$
g_{s}(\mathbf{D})=\max \left[\frac{2 \sigma_{x x}^{2}+6\left(\sigma_{x \eta}^{2}+\sigma_{x \zeta}^{2}\right)}{6}-\frac{\sigma_{\text {allowable }}^{2}}{3}\right] \leq 0
$$

where max[] denotes the maximum value of Eq. (8) over each set of azimuth angle and blade station at which it is evaluated. Therefore, the stress constraint is enforced at the blade station and azimuth angle at which the stress condition is most critical. The stress margin is given by

$$
1-\frac{\sqrt{\sigma_{x x}^{2}+3\left(\sigma_{x \eta}^{2}+\sigma_{x \zeta}^{2}\right)}}{\sigma_{\text {allowable }}}
$$

A stress margin $<0$ would correspond to a design that violates the stress constraint.

\section{Global Approximation Methods (Surrogates)}

To conduct a global search of the design space in a reasonable amount of time, it is necessary to use global approximation, or surrogate methods, in which the true objective function and expensive constraints are replaced with smooth functional relationships that can be evaluated quickly. To construct the surrogates, the objective function and constraints must first be evaluated over a set of design points. The surrogate is then generated by fitting the initial design points. Although function evaluations that come from the expensive helicopter simulations are needed to form the approximation, the initial investment of computer time is significantly less compared with global searches using nonsurrogate-based optimization methods. Once the surrogates have been obtained, they are used to replace the more expensive true objective function and constraints in the search for the optimum.

The surrogate vibration objective function can be generated in two ways:

1) The underlying responses [i.e., the vibratory-hub shears and moments in Eq. (1)] are replaced by surrogates that are used to build the surrogate objective function as in Eq. (11).

2) The overall output $J$ is approximated directly.

Six responses need to be approximated in the first approach, and one response needs to be approximated in the second approach. Both methods were considered in this study:

$$
\begin{aligned}
\hat{J}= & K_{S} \sqrt{\left(\hat{F}_{4 X}\right)^{2}+\left(\hat{F}_{4 Y}\right)^{2}+\left(\hat{F}_{4 Z}\right)^{2}} \\
& +K_{M} \sqrt{\left(\hat{M}_{4 X}\right)^{2}+\left(\hat{M}_{4 Y}\right)^{2}+\left(\hat{M}_{4 Z}\right)^{2}}
\end{aligned}
$$

The stress constraint is the only constraint that requires a forwardflight simulation and is therefore the only computationally expensive constraint. Consequently, a surrogate constraint is used in place of Eq. (9) during optimization. Descriptions of the methods for constructing the global approximations are given next.

\section{A. Design of Computer Experiments}

The space-filling [7] design of computer experiment employed in this study is optimal Latin hypercube (OLH) sampling, which is a commonly used method. The OLH algorithm from the iSIGHT software package is used [21-23]. Methods for fitting the data points in the OLH are described next.

\section{B. Polynomial Response Surfaces}

Suppose that a deterministic function of $N_{\mathrm{dv}}$ design variables needs to be approximated and has been evaluated at $N_{\mathrm{sp}}$ sample points. Sample point $i$ is denoted as $\mathbf{x}^{(i)}=\left(x_{1}^{(i)}, \ldots, x_{N_{\mathrm{dv}}}^{(i)}\right)$ and the associated response is given by $y_{i}=y\left(\mathbf{x}^{(i)}\right)$ for $i=1, \ldots, N_{\mathrm{sp}}$. Note that $\mathbf{x}^{(i)}$ is a scaled version of $\mathbf{D}$ such that its elements vary between 0 and 1 . A polynomial regression approximation to $y(\mathbf{x})$ can be written as

$$
y(\mathbf{x})=\hat{y}(\mathbf{x})+\epsilon_{\mathrm{pr}}
$$

where $\hat{y}(\mathbf{x})$ is the function chosen to approximate the true response $y(\mathbf{x})$, and $\epsilon_{\mathrm{pr}}$ is the error associated with the approximation. It is important to note that the errors are assumed to be independent; that is, the errors at two points close together will not necessarily be close. This assumption will be revisited when considering kriging. In this study, second-order polynomials are used for $\hat{y}(\mathbf{x})$. The least-squares regression approximation is given as [24]

$$
\hat{y}_{\text {poly }}=\beta_{0}+\sum_{i=1}^{N_{\mathrm{dv}}} \beta_{i} x_{i}+\sum_{i=1}^{N_{\mathrm{dv}}} \sum_{j=1, i<j}^{N_{\mathrm{dv}}} \beta_{i j} x_{i} x_{j}+\sum_{i=1}^{N_{\mathrm{dv}}} \beta_{i i} x_{i}^{2}
$$

In addition to Eq. (13), a reduced-term polynomial surrogate in which statistically insignificant terms are removed is considered. The 
reduced-term polynomial is obtained by sequentially removing coefficients with $t$ statistics less than 1 from the full-term polynomial.

\section{Kriging Surrogates}

Kriging is based on the fundamental assumption that errors are correlated, which is in contrast to the assumption of independent or uncorrelated errors made in polynomial regression. This implies that one assumes that the errors at two points close together will be close. In fact, the assumption that the errors are uncorrelated is only appropriate when the sources of error are random, such as in the case of measurement error or noise. In the case of deterministic computer simulations, there is no source of random error. Therefore, it is more reasonable to assume that the error terms will be correlated and the closer that two points are to each other, the higher this correlation will be.

In kriging, the unknown function $y(\mathbf{x})$ is assumed to be of the form

$$
y(\mathbf{x})=f(\mathbf{x})+Z(\mathbf{x})
$$

where $f(\mathbf{x})$ is an assumed function (usually polynomial form), and $Z(\mathbf{x})$ is a realization of a stochastic (random) process that is assumed to be Gaussian [25]. The function $f(\mathbf{x})$ can be thought of as a global approximation of $y(\mathbf{x})$, and $Z(\mathbf{x})$ accounts for local deviations that ensure that the kriging model interpolates the data points exactly. In this study, $Z(\mathbf{x})$ is based on Gaussian spatial correlation functions, and $f(\mathbf{x})$ is assumed to be a linear polynomial. Maximum likelihood estimation is used to select the fitting parameters [26,27]. The kriging surrogates were created with a freely available $\bar{M}$ ATLAB kriging toolbox [28].

\section{Radial Basis Neural Networks}

Radial basis neural networks (RBNNs) approximate a function as a weighted sum of radial basis functions, also known as neurons:

$$
\hat{y}_{\mathrm{RBNN}}=\sum_{i=1}^{N_{\mathrm{RBF}}} \alpha_{i} \phi_{\mathrm{RBNN}}(\mathbf{x})
$$

where $\phi_{\mathrm{RBNN}}(\mathbf{x})$ is the response of the radial basis function at $\mathbf{x}$, and $\alpha_{i}$ is the weight associated with the radial basis function. In this study, the MATLAB routine newrb is used to construct the RBNN. Gaussian functions given by Eq. (16) are used for the neurons:

$$
\phi_{\mathrm{RBNN}}(\eta)=\exp \left(-\eta^{2}\right)
$$

In this case, the dummy variable $\eta$ would be $\left(\tau\left\|\mathbf{x}-\mathbf{x}^{(i)}\right\|\right)$, where $\left\|\mathbf{x}-\mathbf{x}^{(i)}\right\|$ is the Euclidean distance between two vectors. The parameter $\tau$ is inversely related to the user-defined spread parameter, which controls the radius of influence for each neuron. Specifically, the radius of influence is the distance at which the output of a neuron reaches a certain small value corresponding to half of the spread parameter. A high spread would cause the neuron responses to be smooth, and a low spread would result in highly nonlinear responses. The spread is set to 0.5 in this study. The number of radial basis functions and associated weights are determined by satisfying the user-defined error goal for the mean square error in the approximation. The goal parameter is set to the square of $5 \%$ of the mean response in this study.

\section{E. Weighted-Average Surrogates}

Once the individual surrogates have been generated, an extra model can be constructed with little additional expense, compared with the cost of generating the fitting data. The additional surrogate is based on the weighted-average approach implemented in [10], in which it was shown that the weighted-average surrogate protected against the worst individual surrogate while performing as well as the best for a number of analytical and applied problems. The weightedaverage surrogate is formulated as a weighted sum of the three individual approximation methods; that is,

$$
\hat{y}_{\mathrm{WTA}}=w_{\text {poly }} \hat{y}_{\text {poly }}+w_{\text {krg }} \hat{y}_{\text {krg }}+w_{\text {RBNN }} \hat{y}_{\text {RBNN }}
$$

where $w_{\text {poly }}, w_{\mathrm{krg}}$, and $w_{\mathrm{RBNN}}$ are the weights associated with each surrogate. The weights are calculated in such a way that they 1) reflect the confidence in each individual surrogate and 2) filter out adverse effects associated with individual surrogates that represent the sample data well, but predict poorly at designs that are not included in the sample data. Furthermore, the weights in Eq. (17) are constrained to sum to 1 so that if all of the individual surrogates give the same output at some input, then the weighted surrogate will also recover this output. A weight scheme that satisfies these issues is given next [10]:

$$
w_{i}=\frac{w_{i}^{*}}{\sum_{i}^{N_{\mathrm{sm}}} w_{i}^{*}}
$$

where

$$
\begin{gathered}
w_{i}^{*}=\left(E_{i}+d_{1} E_{\text {avg }}\right)^{d_{2}}, \quad d_{1}<1, d_{2}<0 \\
E_{\text {avg }}=\sum_{i}^{N_{\mathrm{sm}}} E_{i} / N_{\mathrm{sm}}
\end{gathered}
$$

and $N_{\mathrm{sm}}$ is the number of surrogate models. The weights are based on a global-data-based error measure for each surrogate, denoted by $E_{i}$. In this study, the generalized mean square error (GMSE) based on leave-one-out cross validation is used as the error measure, and thus

$$
E_{i}=\sqrt{\mathrm{GMSE}_{i}}
$$

Details on how the GMSE is determined are given in [10]. In Eq. (19), $d_{1}$ and $d_{2}$ are user-defined parameters that control the relative influence of the individual surrogate error $E_{i}$ and the average of the individual errors $E_{\text {avg }}$ on the weight. Small values of $d_{1}$ and large negative values of $d_{2}$ result in high weights for the best individual surrogate, which satisfies the first goal mentioned previously for determining the weights. Large values of $d_{1}$ and small negative values of $d_{2}$ result in more emphasis on the average of the error, which would protect against surrogates that may predict well at sample data points, but predict poorly at unsampled locations in the design space. Based on a parametric study conducted in [10], setting $d_{1}=0.05$ and $d_{2}=-1$, or $d_{1}=0.5, d_{2}=-1$ has little effect on the weights. Because similar behavior was observed in this study, the results are presented for $d_{1}=0.05$ and $d_{2}=-1$. Note that the intuitive property that the higher the error, the lower the weight corresponding to a surrogate is recovered because $d_{2}<0$. It is worth noting that the optimal settings of $d_{1}$ and $d_{2}$ as well as the optimal choice of surrogates for use in the weighted-average approach are important issues that are the subject of ongoing research beyond the scope of this paper.

\section{Results}

This section presents accuracy measures of the approximation methods that have been described and vibration-reduction results using the surrogate objective functions. The helicopter configuration used in all computations is given in Table 1. The simulations are conducted at an advance ratio of 0.15 and descent angle of $6.5 \mathrm{deg}$, where high vibration levels due to strong BVI are encountered. Optimization results are compared with a baseline design resembling a Messerschmitt-Bölkow-Blohm (MBB) BO-105 blade. Figure 3 illustrates the geometrical data needed for the propulsive-trim calculation used in this study.

In addition to the information provided in Table 1, additional information is needed for the fixed cross-sectional parameters, objective function, constraints, and finite element discretization of the blade. The material properties and the chordwise locations of the vertical walls are given in Table 2 .

The weighting factors in the objective function, $K_{S}$ and $K_{M}$, are selected to be 1 . These weighting factors result in an objective function that represents the sum of the $4 /$ rev oscillatory-hub shear resultant and the 4/rev oscillatory-hub moment resultant in the hubfixed nonrotating frame. For this study, the following side constraints 
Table 1 Rotor and helicopter parameters

\begin{tabular}{lc}
\hline \hline \multicolumn{3}{c}{ Dimensional data } \\
$R=4.91 \mathrm{~m}$ & $\Omega=425 \mathrm{rpm}$ \\
$m_{0}=5.57 \mathrm{~kg} / \mathrm{m} \quad$ Nondimensional data \\
$N_{b}=4 \quad c=0.05498 R$ \\
$\beta_{p}=0.0 \mathrm{deg}$ & $C_{d 0}=0.01$ \\
$\theta_{\mathrm{pt}}=0 \mathrm{deg}$ & $\alpha_{\mathrm{d}}=6.5 \mathrm{deg}$ \\
$\mu=0.15$ & $C_{W}=0.005$ \\
$\sigma=0.07$ & $C_{d f}=0.01$ \\
$X_{F A}=0.0$ & $Z_{F A}=0.3$ \\
$X_{F C}=0.0$ & $Z_{F C}=0.3$ \\
$M B B B O-105$ & baseline fundamental frequencies \\
$\omega_{L 1}=0.729$ & $\omega_{F 1}=1.125$ \\
$\omega_{T 1}=3.263$ & \\
\hline \hline
\end{tabular}

are enforced:

$$
\begin{gathered}
1.0 \mathrm{~mm} \leq t_{1} \leq 8.0 \mathrm{~mm} \\
1.0 \mathrm{~mm} \leq t_{2}, t_{3} \leq 12.0 \mathrm{~mm} \\
0.0 \leq m_{\mathrm{ns}} / m_{0} \leq 0.25
\end{gathered}
$$

The upper and lower bounds used for the frequency-placement constraints, per revolution, are given next:

$$
\begin{aligned}
& 0.60 \leq \omega_{L 1} \leq 0.80 \\
& 1.05 \leq \omega_{F 1} \leq 1.20 \\
& 2.50 \leq \omega_{T 1} \leq 6.50
\end{aligned}
$$

In the aeroelastic stability constraints given by Eq. (7), the minimum acceptable damping for all modes, $\left(\zeta_{k}\right)_{\min }$, is chosen to be 0.01 . Additionally, the constraints are modified for the second lag mode, which can sometimes be slightly unstable. To prevent this situation, a small amount of structural damping is added to this mode. For this study, $0.5 \%$ structural damping is added to stabilize the second lag mode of the baseline blade. For the stress constraint, a factor of safety of 1.5 is used. The rotor blade was discretized into the 6 finite elements, shown in Fig. 4.

In this study, two sets of fitting points are used to build the surrogates: a 300-point OLH and a 500-point OLH. From the 300point OLH, 283 points had converged-trim solutions and were used to build the surrogates, whereas out of the 500 -point OLH, 484 points had converged-trim solutions. One of the advantages of surrogatebased optimization with the design of computer experiments is that each simulation corresponding to a design point in the OLH can be run independently of the others, and therefore the simulations can be run in parallel. The helicopter simulations were run on a Linux

\begin{tabular}{|c|c|c|}
\hline & Parameters & Values \\
\hline \multicolumn{3}{|c|}{ Aluminum material properties } \\
\hline$E$ & & $70.7 \mathrm{GPa}$ \\
\hline$v$ & & 0.33 \\
\hline$\rho_{\text {struct }}$ & & $2700 \mathrm{~kg} / \mathrm{m}^{3}$ \\
\hline$\sigma_{Y}$ & & $324 \mathrm{MPa}$ \\
\hline \multicolumn{3}{|c|}{ Nonstructural filler mass density } \\
\hline$\rho_{\text {filler }}$ & & $237.4 \mathrm{~kg} / \mathrm{m}^{3}$ \\
\hline \multicolumn{3}{|c|}{ Locations of the vertical walls } \\
\hline$x_{1}$ & & $65.4 \mathrm{~mm}$ \\
\hline$x_{2}$ & & $111.6 \mathrm{~mm}$ \\
\hline
\end{tabular}
cluster of 1.8-2.4 GHz Opteron processors. Using 40 processors, the

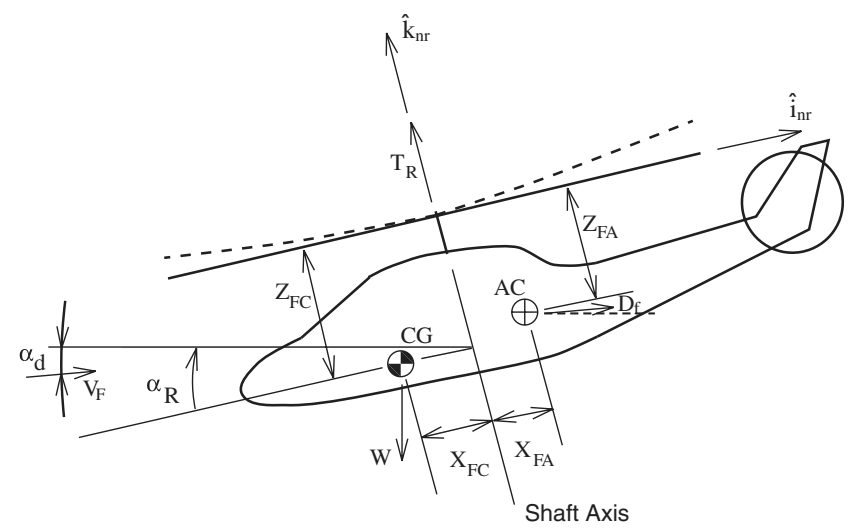

Fig. 3 Helicopter in forward flight.
Table 2 Fixed parameters defining the blade cross section

283-point data set required $53 \mathrm{~h}$ to generate, and the 484-point data set needed $82 \mathrm{~h}$.

The fitting times associated with each of the approximation methods considered in this study are presented in Table 3 . Note that the abbreviation (red) indicates the use of reduced-term polynomial surrogates. The individual surrogates were generated on $3.2 \mathrm{GHz}$ Xeon processors, and the 40 processors from the Linux cluster were used to generate the weighted-average models. The leave-one-out cross-validation error needed to generate the weighted models is suitable for parallel computation because the error at left-out points can be calculated independently of the errors at the other points. These results demonstrate that constructing the surrogates in this study, including weighted-average models, required little additional time, compared with the time needed to generate the fitting data.

\section{A. Weighted-Average Surrogate Construction}

The weight coefficients necessary to define the weighted-average surrogates are given in Tables $\underline{4}$ and $\underline{5}$. The weight coefficients obtained when using the full-term polynomial response surface are given in Table $\underline{4}$. Generally, the kriging surrogate has the highest weight for all responses and both sample sizes in Table 4 . When the reduced-term polynomial is used in place of the full-term polynomial, as shown in Table $\underline{5}$, the polynomial is weighted the most for all responses and sample sizes. The RBNN generally has the lowest weight. Because the RBNN corresponds to the highest leaveone-out cross-validation errors, their responses are the most sensitive to the individual data points used to fit the model. This suggests that the poor performance of the RBNN is due to overfitting of the data.

\section{B. Surrogate Accuracy Results}

The predictive capabilities of the individual and weighted-average surrogates were quantified using a set of data points not included in the construction of the surrogates. The predicted responses from the surrogates were then compared with the actual responses at the test points. The test points came from a 200-point OLH, of which $197 \mathrm{had}$ converged-trim solutions. None of the blade designs from the 197 test points were coincident with the blade designs from the two OLHs used to create the surrogates. Using the test points, the absolute percent error is given by

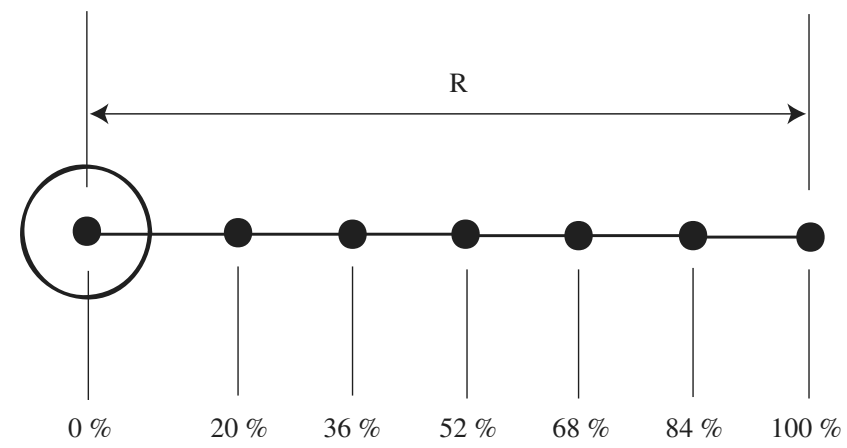

Fig. 4 Finite element node locations. 
Table 3 Fitting times associated with the approximation methods

\begin{tabular}{lcc}
\hline \hline Surrogate & sample size $=$ & Fitting time \\
\hline Polynomial & 283 & $<1 \mathrm{~s}$ \\
Polynomial (red) & 283 & $<1 \mathrm{~s}$ \\
Kriging & 283 & $15-20 \mathrm{~s}$ \\
RBNN & 283 & $30-50 \mathrm{~s}$ \\
Weighted average & 283 & $7-10 \mathrm{~min}$. \\
Weighted average (red) & 283 & $7-10 \mathrm{~min}$. \\
Polynomial & 484 & $<1 \mathrm{~s}$ \\
Polynomial (red) & 484 & $1-2 \mathrm{~s}$ \\
Kriging & 484 & $50 \mathrm{~s}-1 \mathrm{~min}$. \\
RBNN & 484 & $1.5-2 \mathrm{~min}$. \\
Weighted average & 484 & $30-40 \mathrm{~min}$. \\
Weighted average (red) & 484 & $30-40 \mathrm{~min}$. \\
\hline \hline
\end{tabular}

${ }^{\text {aThe }} 283$ and 484 sample points required 53 and $82 \mathrm{~h}$ to generate, respectively.

$$
\varepsilon_{i}=\frac{\left|y^{(i)}-\hat{y}^{(i)}\right|}{\bar{y}}
$$

where $y^{(i)}$ is the actual response computed by the helicopter simulation, and $\hat{y}^{(i)}$ is the response predicted by the surrogate at the $i$ th test point. For the vibratory-load errors, $\bar{y}$ is the mean of the absolute values of the responses from the 197 test points, and for the errors in the surrogate stress constraint,

$$
\bar{y}=\frac{\sigma_{\text {allowable }}^{2}}{3}
$$

Based on Eq. (28), the average error is

$$
\varepsilon_{\mathrm{avg}}=\frac{\sum_{i=1}^{N_{\mathrm{tp}}} \varepsilon_{i}}{N_{\mathrm{tp}}}
$$

where $N_{\mathrm{tp}}$ is the number of test points. The average percent error is representative of the surrogate's predictive capability over the entire design space, because all 197 test points are included.

The average errors are given in Fig. 5 for 7 responses: the 6 underlying hub shears and moments and the stress constraint. For the 283-point sample set, the kriging surrogate has the lowest average error of the individual surrogates for each response. In contrast, the polynomials correspond to the lowest average errors for some responses based on the 484-point sample set. So the choice of the best surrogate in terms of approximating over the entire design space is dependent on the sample size for the responses considered in this study. If one were to only use kriging with larger sample sets (e.g., the 484 fitting points) because it was the most accurate method with 283 points, instead of continuing to use multiple surrogates, then inferior results would be obtained. Furthermore, given that constructing various surrogates is relatively inexpensive, there is no penalty associated with taking advantage of multiple approximation methods, even those that may have performed poorly under certain circumstances.

Among the individual surrogates for the 7 responses shown in Fig. 5, the lowest average errors range from 2-53\% for the 283-point sample set. In comparison, the errors of the weighted-average surrogates range from $2-53 \%$ and always correspond to lower errors than the worst approximation method. These results demonstrate that the weighted-average surrogates performed as well as the best individual surrogates for the responses considered in this study.

The errors in the surrogate objective function are given in Fig. 6 for both methods of forming the approximate objective function. A comparison of the errors in Figs. 5 and 6 with the weight coefficients in Tables $\underline{4}$ and $\underline{5}$ shows that the surrogate with the lowest crossvalidation error (and hence the highest weight) is not necessarily the most accurate when using test points to measure error. For example, consider the case of approximating the overall objective function directly. The results in Table 5 indicate that reduced-term polynomials are more accurate than kriging surrogates when using the GMSE as the global error measure. However, Fig. $\underline{6}$ shows that the kriging surrogates are more accurate than the reduced-term polynomials in terms of the average error based on test points. This example illustrates a potential downside associated with attempting to identify the most accurate individual surrogate for a given application: the most accurate approximation method may be dependent on the metric used to quantify error.

It is interesting to note that increasing the sample size from 283 to 484 generally had little effect on the accuracy of the surrogates. This indicates that for the 17-dimensional design space, increasing the number of fitting points from 283 to 484 was not sufficient to significantly enhance the surrogates' predictions for the responses considered in this study.

\section{Optimization Results}

Optimization results based on surrogate objective-functions and constraints are presented in this section. Optimization of the surrogate objective functions was conducted with the multi-island genetic algorithm in iSIGHT [29]. The genetic algorithm was set to run for 200,000 total objective-function evaluations. Optimization of each surrogate objective function was conducted simultaneously because they can be optimized in parallel.

Table $\underline{6}$ gives the optimization results when using the underlying hub shears and moments to build the surrogate objective function. Note that vibration-reduction results are presented relative to the

Table 4 Weight coefficients for the weighted-average surrogates with full-term polynomials

\begin{tabular}{lccccccccc}
\hline \hline Weight coefficient & Sample size & $F_{4 X}$ & $F_{4 Y}$ & $F_{4 Z}$ & $M_{4 X}$ & $M_{4 Y}$ & $M_{4 Z}$ & $J$ & Stress constraint \\
\hline$w_{\text {poly }}$ & 283 & 0.407 & 0.395 & 0.322 & 0.374 & 0.306 & 0.291 & 0.333 & 0.353 \\
$w_{\text {krg }}$ & 283 & 0.478 & 0.473 & 0.458 & 0.460 & 0.449 & 0.412 & 0.461 & 0.443 \\
$w_{\text {RBNN }}$ & 283 & 0.115 & 0.132 & 0.219 & 0.167 & 0.245 & 0.297 & 0.206 & 0.205 \\
$w_{\text {poly }}$ & 484 & 0.422 & 0.436 & 0.360 & 0.378 & 0.340 & 0.333 & 0.379 & 0.400 \\
$w_{\text {krg }}$ & 484 & 0.449 & 0.422 & 0.425 & 0.448 & 0.405 & 0.381 & 0.419 & 0.424 \\
$w_{\text {RBNN }}$ & 484 & 0.129 & 0.142 & 0.215 & 0.175 & 0.255 & 0.286 & 0.203 & 0.176 \\
\hline \hline
\end{tabular}

Table 5 Weight coefficients for the weighted-average surrogates with reduced-term polynomials

\begin{tabular}{lccccccccc}
\hline \hline Weight coefficient & Sample size & $F_{4 X}$ & $F_{4 Y}$ & $F_{4 Z}$ & $M_{4 X}$ & $M_{4 Y}$ & $M_{4 Z}$ & $J$ & Stress constraint \\
\hline$w_{\text {poly }}$ & 283 & 0.497 & 0.493 & 0.431 & 0.472 & 0.406 & 0.401 & 0.443 & 0.433 \\
$w_{\text {krg }}$ & 283 & 0.406 & 0.396 & 0.385 & 0.388 & 0.385 & 0.349 & 0.385 & 0.388 \\
$w_{\text {RBNN }}$ & 283 & 0.097 & 0.110 & 0.183 & 0.140 & 0.209 & 0.250 & 0.172 & 0.179 \\
$w_{\text {poly }}$ & 484 & 0.465 & 0.474 & 0.409 & 0.427 & 0.384 & 0.377 & 0.424 & 0.436 \\
$w_{\text {krg }}$ & 484 & 0.415 & 0.394 & 0.393 & 0.413 & 0.378 & 0.356 & 0.388 & 0.399 \\
$w_{\text {RBNN }}$ & 484 & 0.120 & 0.132 & 0.199 & 0.161 & 0.238 & 0.267 & 0.188 & 0.165 \\
\hline \hline
\end{tabular}




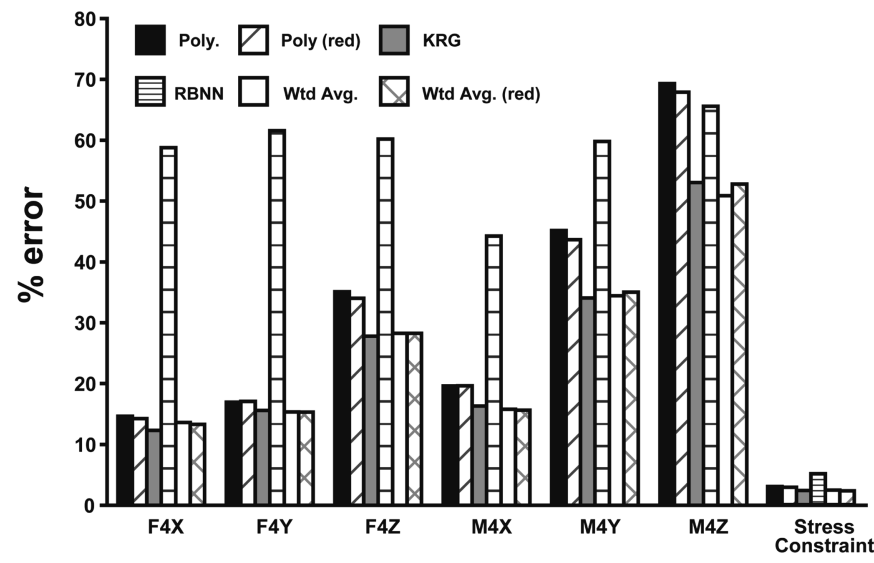

a) 283 sample points

verage errors of the underlying vibratory loads and stress constraint (Poly. denotes the polynomial, KRG denotes kriging, and Wtd. Avg. denotes the weighted average).

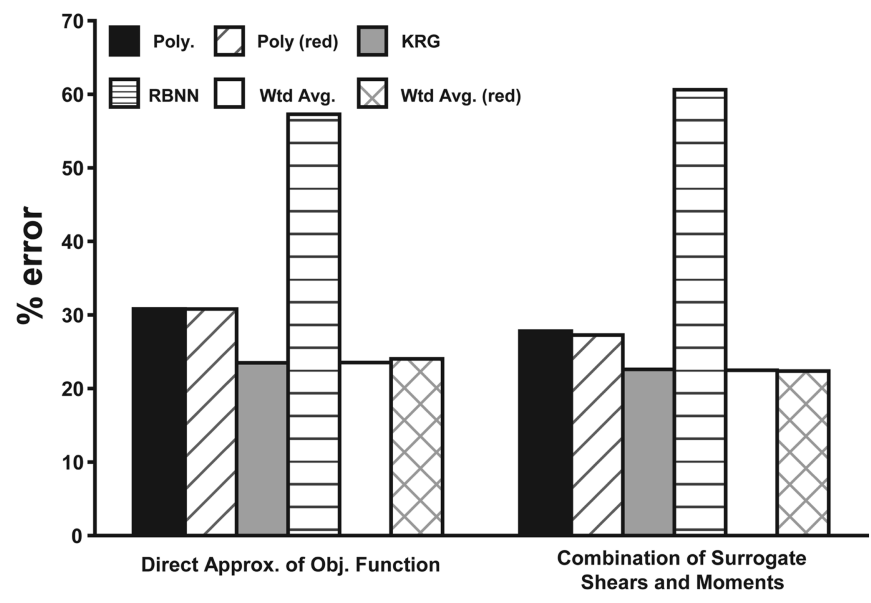

a) 283 sample points

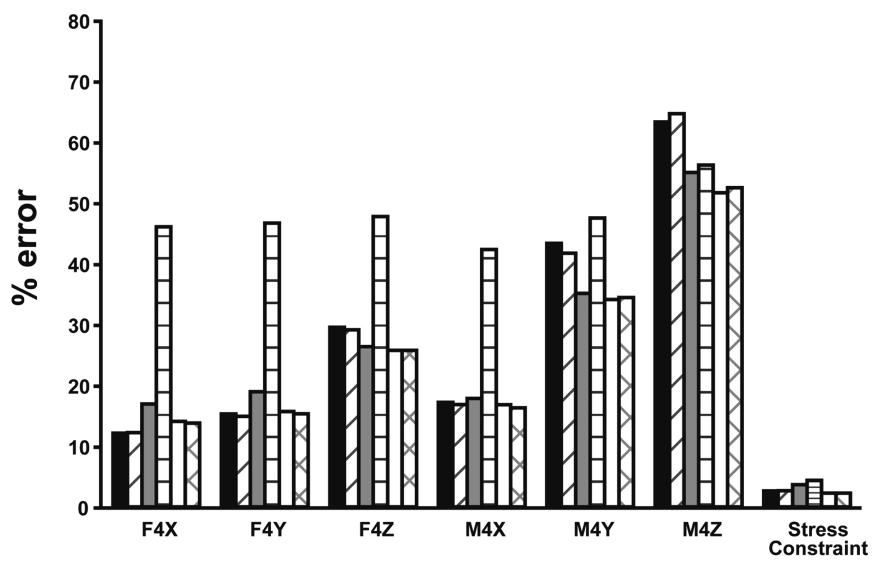

b) 484 sample points 
Table 7 Optimization results when directly approximating the objective function

\begin{tabular}{|c|c|c|c|c|c|c|c|c|}
\hline Surrogate & Sample size & Optimization time, $\mathrm{h}$ & Predicted reduction & Actual reduction & Actual stress margin & $\omega_{L 1}$ & $\omega_{F 1}$ & $\omega_{T 1}$ \\
\hline Polynomial & 283 & 2 & $394.4 \%$ & $64.4 \%$ & 0.005 & 0.610 & 1.058 & 4.330 \\
\hline Polynomial (red) & 283 & 2 & $512.3 \%$ & $60.1 \%$ & 0.005 & 0.605 & 1.057 & 4.231 \\
\hline Kriging & 283 & 2 & $120.0 \%$ & $54.1 \%$ & 0.006 & 0.600 & 1.055 & 4.252 \\
\hline RBNN & 283 & 4 & $93.9 \%$ & $57.4 \%$ & 0.009 & 0.602 & 1.055 & 4.420 \\
\hline Weighted average & 283 & 4 & $234.9 \%$ & $70.5 \%$ & 0.008 & 0.604 & 1.055 & 4.538 \\
\hline Weighted average (red) & 283 & 4 & $221.7 \%$ & $65.0 \%$ & 0.000 & 0.604 & 1.059 & 3.871 \\
\hline Polynomial & 484 & 2 & $222.4 \%$ & $45.0 \%$ & 0.001 & 0.627 & 1.060 & 3.960 \\
\hline Polynomial (red) & 484 & 2 & $207.1 \%$ & $50.0 \%$ & 0.000 & 0.600 & 1.058 & 3.710 \\
\hline Kriging & 484 & 2 & $145.1 \%$ & $55.8 \%$ & 0.000 & 0.606 & 1.057 & 3.981 \\
\hline RBNN & 484 & 4 & $97.4 \%$ & $67.5 \%$ & 0.000 & 0.631 & 1.057 & 4.670 \\
\hline Weighted average & 484 & 4 & $116.7 \%$ & $67.6 \%$ & 0.010 & 0.620 & 1.057 & 4.602 \\
\hline Weighted average (red) & 484 & 4 & $129.2 \%$ & $58.8 \%$ & 0.008 & 0.625 & 1.056 & 4.380 \\
\hline
\end{tabular}

Table 8 Predicted vibration reduction and stress margins by each of the surrogates when directly approximating the objective function

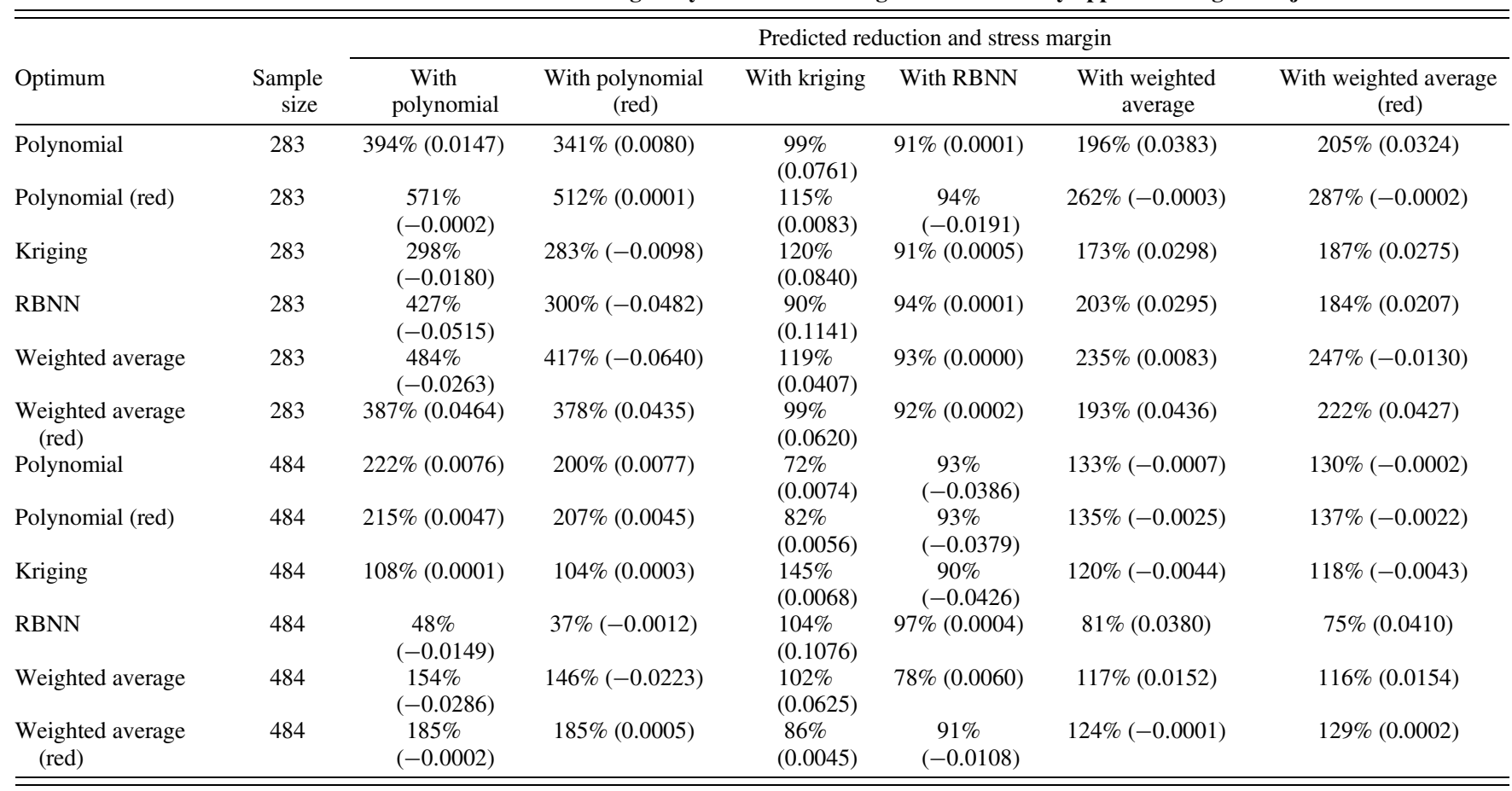

additional hours, which is relatively insignificant compared with the $53 \mathrm{~h}$ needed to generate the 283 sample points. Similar results for the 484-point sample set are clear from Table 6.

Optimization results corresponding to direct approximation of the overall objective function are provided in Table 7 . The average, maximum, and minimum Euclidean distances relative to the maximum dimension of the design space are 33, 51, and $18 \%$, respectively, for the 283-point data set and 33, 43, and $18 \%$, respectively, for the 484-point sample set. The full-term polynomials led to the most vibration reduction among the individual surrogates based on the 283 sample points, but led to the worst design with the 484 fitting points. The RBNN, which is the least accurate surrogate, led to the best design for the 484-point sample set. These results represent extreme examples in the sense that the best individual surrogate for one sample set is the worst surrogate for another sample set, and the least accurate surrogate led to the best design in one instance. Thus, optimizing the least accurate surrogate proved to be beneficial. Because it is relatively inexpensive to optimize the surrogates, there is no downside to considering all available surrogates for optimization, including the approximation method that performs the worst under some circumstances. Furthermore, the weighted-average surrogates led to the lowest-vibration designs in Tables 6 and 7. Therefore, it was beneficial to optimize the weightedaverage models, because they located promising designs that were overlooked by the individual surrogates.
The different fundamental blade frequencies presented in Tables 6 and 7, along with the Euclidean distances between optimum designs, demonstrate that optimization of multiple surrogates was useful for locating distinct reduced-vibration designs. In addition to employing multiple approximation methods, considering both methods of constructing the surrogate objective function resulted in additional

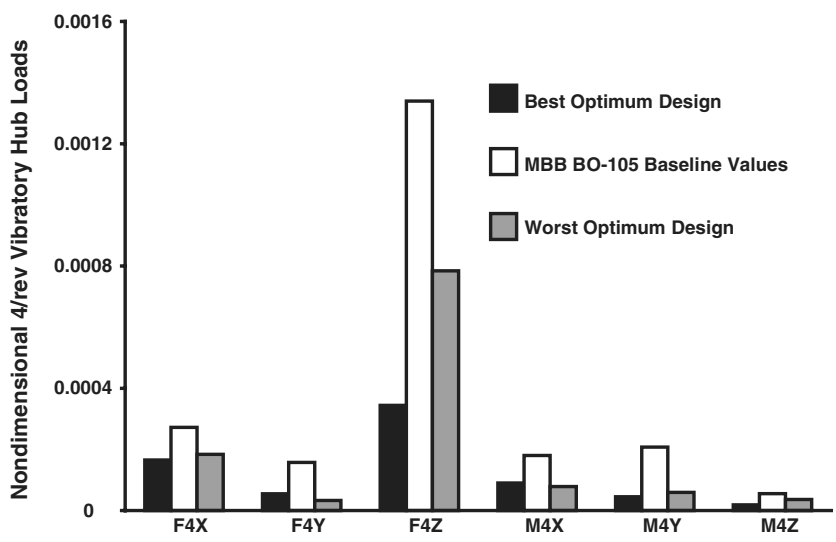

Fig. 7 Comparison of the best and worst designs from Tables $\underline{6}$ and $\underline{7}$ with the baseline design. 
reduced-vibration designs. For instance, the best design associated with constructing the surrogate objective function from the underlying responses corresponds to a $70.2 \%$ vibration reduction, and the best design from direct approximation results in a $70.5 \%$ reduction. The differences in the fundamental frequencies in Tables $\underline{6}$ and $\underline{7}$ demonstrate that these are two distinct designs, even though they correspond to similar levels of vibration reduction. Thus, given that the 24 surrogate objective functions in Tables $\underline{6}$ and $\underline{7}$ were optimized simultaneously using parallel computation, a multiplesurrogate approach was an inexpensive and effective method for

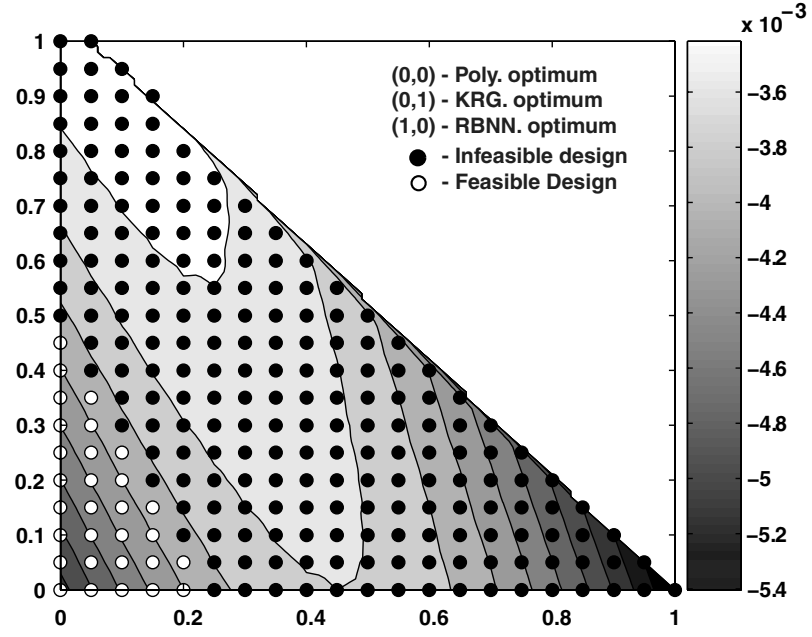

a) Polynomial

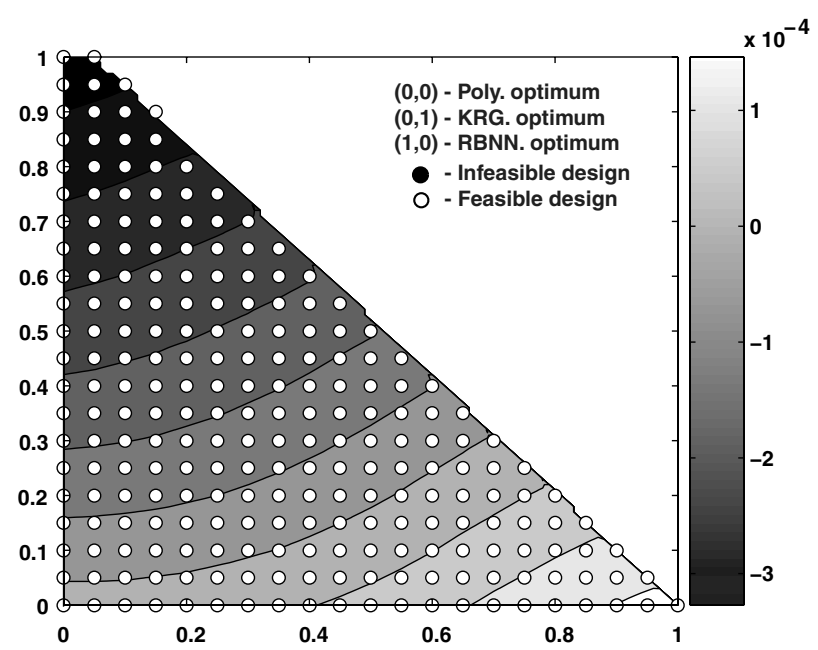

c) Kriging

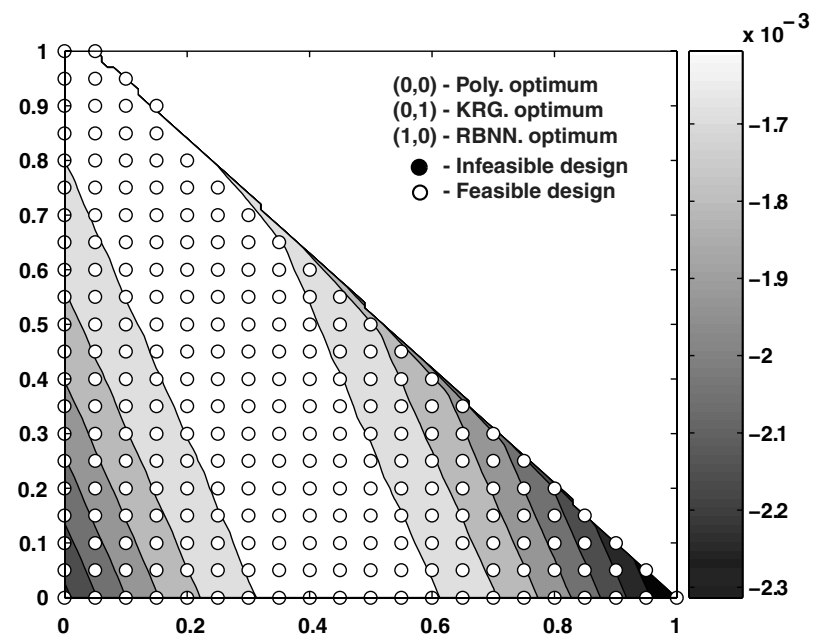

e) Weighted average

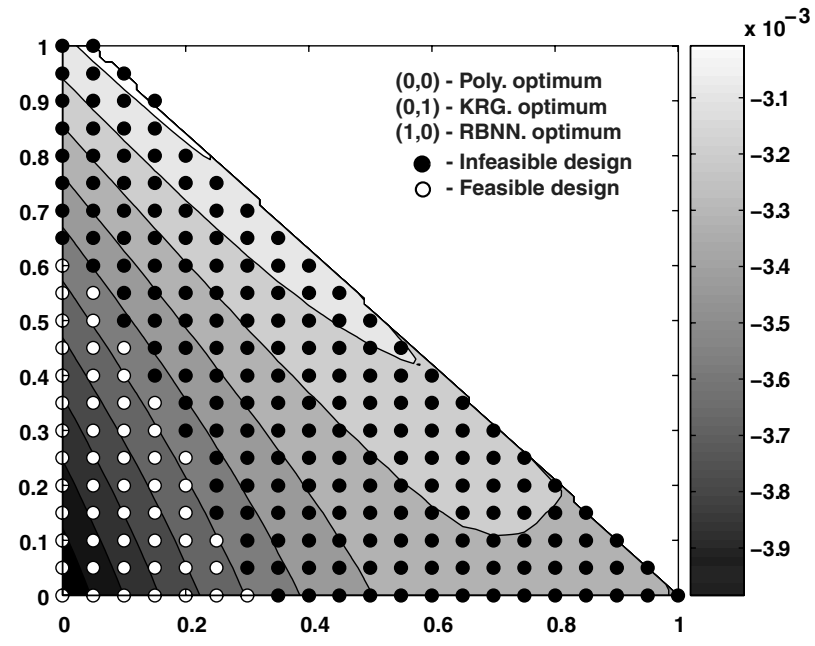

b) Polynomial (red)

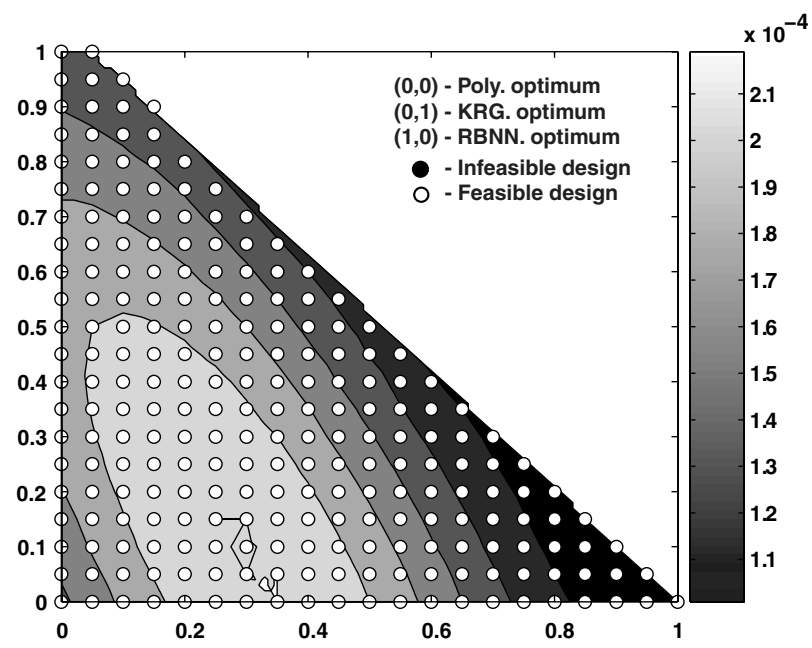

d) RBNN

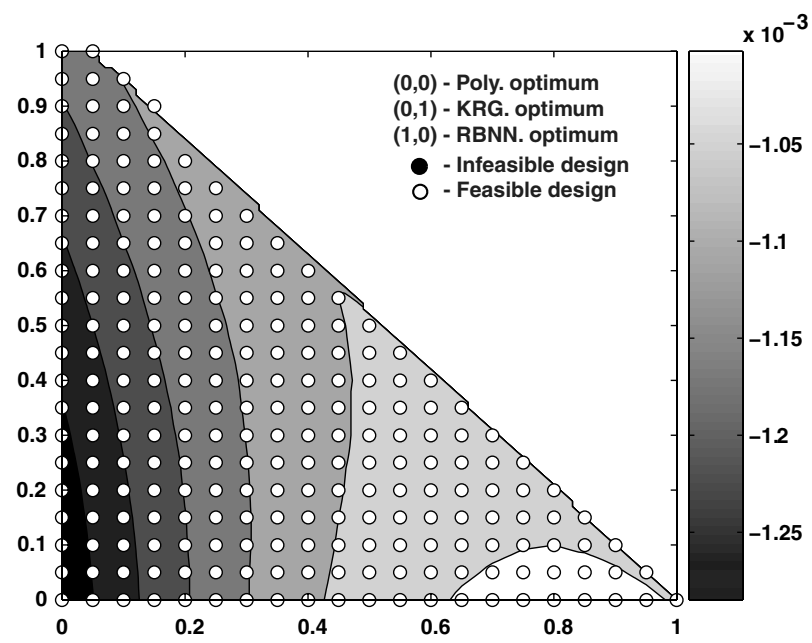

f) Weighted average (red)

Fig. 8 Surrogate objective-function contours plotted in a plane defined by the optimum designs corresponding to full-term polynomials, kriging, and RBNNs based on 283 sample points; evaluations of the surrogate stress constraint at various designs in the plane are represented by circles. 
locating various candidate designs. Using multiple surrogates decreases the likelihood of overlooking promising designs, because different surrogates may favor different regions of the design space.

Although all of the optimum designs in Tables $\underline{6}$ and $\underline{7}$ are different, they all result in significant reduction of the vertical shear $F_{4 Z}$, which is the primary mechanism for reducing the objective function corresponding to the BVI flight condition. This is illustrated in Fig. 7, in which the best and worst designs from Tables 6 and 7 (70.5 and $45.0 \%$ vibration reduction, respectively) are compare $\bar{d}$ with the MBB BO-105 baseline vibratory loads. These two designs reduce the vertical shear by 74 and $41 \%$. Although the other vibratory loads are also reduced, the vertical shear is the largest and therefore its reduction is the most critical for minimizing the objective function. Furthermore, all of the designs in Tables 6 and 7 correspond to stress margins less than 0.02 , which indicates that the stresses in the optimal blades are relatively close to the allowable stress. Because blade designs corresponding to significant vibration reduction that lie near the allowable-stress boundary were located, it was felt that iterative search methods such as those based on a trustregion strategy [30] or uncertainty in the surrogates [31] were not worth the additional cost.

The significant differences in predicted and actual amounts of vibration reduction in Tables $\underline{6}$ and $\underline{7}$ indicate that the surrogates are inaccurate at their respective optimal designs. Furthermore, the surrogates were susceptible to predicting impractical amounts of vibration reduction (i.e., $\geq 100 \%$ ). Thus, it was especially critical for this problem to conduct simulations at each optimal design to obtain the actual amount of reduction. Even though they were not accurate everywhere in the design space, the surrogates still led to reducedvibration designs.

To illustrate the effects of errors in the individual surrogate constraints on optimization results, the predicted vibration reductions from each surrogate at all of the optimal designs from Table 7 are given in Table 8 , along with predicted stress margins in parentheses. Although all surrogates predict that each optimal design is a reduced-vibration design, some designs were missed due to errors in the surrogate constraints. For example, among the individual approximation methods based on 283 sample points, the full-term polynomial-surrogate objective function predicts that the optimum design corresponding to the RBNN results in a $427 \%$ reduction, which is superior to the $394 \%$ predicted reduction corresponding to its own optimum. However, as indicated by the stress margin, the full-term polynomial-surrogate stress constraint incorrectly predicts that the RBNN optimum design is infeasible. Therefore, errors in the polynomial-surrogate constraint led away from a design that was predicted to correspond to a better objectivefunction value. Figure 8 , which illustrates this result using the visualization method introduced in [32], provides contour plots of the approximate objective functions plotted in a plane defined by three of the optimal designs from the individual surrogates. The minima returned by the full-term polynomial, kriging, and RBNN surrogates are shown as $(0,0),(0,1)$, and $(1,0)$ and define the endpoints on the plane. Circles representing evaluations of the surrogate stress constraint over various designs in the plane are superimposed on the contour plots. For example, Fig. 8a shows that the full-term polynomial predicts that the RBNN optimum corresponds to a superior objective-function value compared with its own optimum. However, Fig. 8a also shows that this design violates the polynomial-surrogate stress constraint.

Table 8 and Fig. 8 also illustrate how the errors in the surrogate objective functions may lead to inferior designs. For example, the kriging-surrogate stress constraint based on 283 sample points correctly predicts that the full-term polynomial design, which is superior to the kriging optimum in terms of actual vibration reduction, is feasible. However, the kriging-surrogate objective function predicts that the superior design corresponds to a $99 \%$ vibration reduction, while predicting a $120 \%$ reduction for the inferior kriging optimum. Therefore, the kriging-surrogate objective function led to an inferior design, compared with the full-term polynomial, because it incorrectly predicted that the kriging optimum was better than the full-term polynomial optimum.

\section{Conclusions}

The results in this paper demonstrate the advantages of employing multiple surrogates as a relatively inexpensive method for fully using expensive sample data. Furthermore, some pitfalls associated with identifying a single surrogate for accurate predictions and/or optimization were exemplified using the rotor blade vibrationreduction problem. The results illustrated that all available surrogates should be optimized, even those that perform poorly under certain circumstances, because there is relatively little cost in doing so. The principal results from this study are summarized next.

1) The rotor blade vibration-reduction problem was illustrative of applications in which there is no single best approximation method. The results indicated that the most accurate approximation method was dependent on sample size and the metric used to quantify error, whereas the best surrogate for optimization differed with sample size.

2) The most accurate approximation method did not always lead to the best design. In fact, the radial basis neural network, which was the least accurate approximation method, led to the best design among all of the individual surrogates when directly approximating the objective function.

3) Optimization of multiple surrogates, including the weightedaverage models, was an effective method for locating reducedvibration blade designs that would have been overlooked if only a single surrogate was employed. Generating the fitting data required 52-82 $\mathrm{h}$ of computation and creating all of the surrogates required less than $1 \mathrm{~h}$, and $8 \mathrm{~h}$ was needed to optimize all of the surrogates based on 200,000 objective-function evaluations each. Feasible designs ranging from a $45-70.5 \%$ vibration reduction were located.

\section{Acknowledgments}

This research was supported in part by the François-Xavier Bagnoud (FXB) Center for Rotary and Fixed Wing Air Vehicle Design, NASA Graduate Student Research Program (GSRP) grant NNA-06CB71H for Bryan Glaz, and in part from the Center for Rotorcraft Innovation (CRI) under work breakdown structure (WBS) 2006-B-01-01.2-A17. Support for Tushar Goel was provided by the Institute for Future Space Transport, under the NASA Constellation University Institute Program (CUIP), with Claudia Meyer as program monitor. Furthermore, some of the material in this study is based upon work supported by the National Science Foundation under grant 0423280.

\section{References}

[1] Friedmann, P. P., "Helicopter Vibration Reduction Using Structural Optimization with Aeroelastic/Multidisciplinary Constraints-A Survey," Journal of Aircraft, Vol. 28, No. 1, Jan. 1991, pp. 8-21. doi: $10.2514 / 3.45987$

[2] Celi, R., "Recent Applications of Design Optimization to RotorcraftA Survey," Journal of Aircraft, Vol. 36, No. 1, Jan.-Feb. 1999, pp. $176-189$.

doi: $10.2514 / 2.2424$

[3] Ganguli, R., "Survey of Recent Developments in Rotorcraft Design Optimization," Journal of Aircraft, Vol. 41, No. 3, May-June 2004, pp. 493-510.

doi: $10.2514 / 1.58$

[4] Friedmann, P. P., and Millott, T. A., "Vibration Reduction in Rotorcraft Using Active Control: A Comparison of Various Approaches," Journal of Guidance, Control, and Dynamics, Vol. 18, No. 4, July-Aug. 1995, pp. 664-673. doi: $10.2514 / 3.21445$

[5] Queipo, N. V., Haftka, R. T., Shy, W., Goel, T., Vaidyanathan, R., and Tucker, P. K., "Surrogate-Based Analysis and Optimization," Progress in Aerospace Sciences, Vol. 41, No. 1, Jan. 2005, pp. 1-28. doi:10.1016/j.paerosci.2005.02.001

[6] Won, K., and Ray, T., "A Framework for Design Optimization Using Surrogates," Engineering optimization, Vol. 37, No. 7, Oct. 2005, pp. 685-703. doi:10.1080/03052150500211911

[7] Simpson, T. W., Booker, A. J., Ghosh, D., Giunta, A. A., Koch, P. N., and Yang, R., "Approximation Methods in Multidisciplinary Analysis and Optimization: A Panel Discussion," Structural and Multi- 
disciplinary Optimization, Vol. 27, No. 5, July 2004, pp. 302-313. doi:10.1007/s00158-004-0389-9

[8] Glaz, B., Friedmann, P. P., and Liu, L., "Surrogate Based Optimization of Helicopter Rotor Blades for Vibration Reduction in Forward Flight," Structural and Multidisciplinary Optimization, Vol. 35, No. 4, Apr. 2008, pp. 341-363. doi:10.1007/s00158-007-0137-z

[9] Zerpa, L., Queipo, N., Pintos, S., and Salager, J., “An Optimization Methodology of Alkaline-Surfactant-Polymer Flooding Process Using Field Scale Numerical Simulation and Multiple Surrogates," Journal of Petroleum Science and Engineering, Vol. 47, Nos. 3-4, June 2005, pp. 197-208. doi:10.1016/j.petrol.2005.03.002

[10] Goel, T., Haftka, R. T., Shyy, W., and Queipo, N. V., "Ensemble of Surrogates," Structural and Multidisciplinary Optimization, Vol. 33, No. 3, Mar. 2007, pp. 199-216. doi:10.1007/s00158-006-0051-9

[11] Viana, F. A. C., Haftka, R. T., Steffen, V., Jr., Butkewitsch, S., and Leal, M. F., "Ensemble of Surrogates: A Framework Based on Minimization of Integrated Square Error," 49th AIAA/ASME/ASCHE/AHS/ASC Structures, Structural Dynamics and Materials Conference, AIAA, Reston, VA, Apr. 2008, pp. 1-27; also AIAA Paper 2008-1885.

[12] Yuan, K. A., and Friedmann, P. P., "Structural Optimization for Vibratory Loads Reduction of Composite Helicopter Rotor Blades with Advanced Geometry Tips," Journal of the American Helicopter Society, Vol. 43, No. 3, July 1998, pp. 246-256.

[13] Myrtle, T. F., and Friedmann, P. P., "Application of a New Compressible Time Domain Aerodynamic Model to Vibration Reduction in Helicopters Using an Actively Controlled Flap," Journal of the American Helicopter Society, Vol. 46, No. 1, Jan. 2001, pp. 32 43.

[14] Patt, D., Liu, L., and Friedmann, P. P., "Rotorcraft Vibration Reduction and Noise Prediction Using a Unified Aeroelastic Response Simulation," Journal of the American Helicopter Society, Vol. 50, No. 1, Jan. 2005, pp. 95-106.

[15] Yuan, K. A., and Friedmann, P. P., "Aeroelasticity and Structural Optimization of Composite Helicopter Rotor Blades with Swept Tips," NASA CR 4665, May 1995.

[16] Johnson, W., CAMRAD/JA-A Comprehensive Analytical Model of Rotorcraft Aerodynamics and Dynamics, Vol. 1Johnson Aeronautics, Palo Alto, CA, 1988.

[17] Johnson, W., CAMRAD/JA-A Comprehensive Analytical Model of Rotorcraft Aerodynamics and Dynamics, Vol. 2, Johnson Aeronautics, Palo Alto, CA, 1988.

[18] Shampine, L. F., Numerical Solution of Ordinary Differential Equations, Chapman and Hall, New York, 1994.

[19] Friedmann, P. P., and Shanthakumaran, P., "Optimum Design of Rotor Blades for Vibration Reduction in Forward Flight," Journal of the American Helicopter Society, Vol. 29, No. 4, 1984, pp. 70-80.

[20] Lim, J. W., and Chopra, I., "Aeroelastic Optimization of a Helicopter Rotor," Journal of the American Helicopter Society, Vol. 34, No. 1,
1989, pp. 55-62.

[21] Morris, M. D., and Mitchell, T. J., "Exploratory Designs for Computer Experiments," Journal of Statistical Planning and Inference, Vol. 43, No. 3, 1995, pp. 381-402.

[22] Jin, R., Chen, W., and Sudjianto, A., "An Efficient Algorithm for Constructing Optimal Design of Computer Experiments," Journal of Statistical Planning and Inference, Vol. 134, No. 1, 2005, pp. 268-287.

[23] Koch, P. N., Evans, J. P., and Powell, D., "Interdigitation for Effective Design Space Exploration Using iSIGHT," Structural and Multidisciplinary Optimization, Vol. 23, No. 2, 2002, pp. 111-126. doi:10.1007/s00158-002-0171-9

[24] Jin, R., Chen, W., and Simpson, T. W., "Comparative Studies of Metamodeling Techniques Under Multiple Modeling Criteria," Structural and Multidisciplinary Optimization, Vol. 23, No. 1, Dec. 2001, pp. 1-13. doi:10.1007/s00158-001-0160-4

[25] Sacks, J., Welch, W. J., Mitchell, T. J., and Wynn, H. P., "Design and Analysis of Computer Experiments," Statistical Science, Vol. 4, No. 4, 1989, pp. 409-435. doi:10.1214/ss/1177012413

[26] Jones, D. R., "A Taxonomy of Global Optimization Methods Based on Response Surfaces," Journal of Global Optimization, Vol. 21, No. 4, Dec. 2001, pp. 345-383. doi:10.1023/A:1012771025575

[27] Sasena, M., "Flexibility and Efficiency Enhancements for Constrained Global Optimization with Kriging Approximations," Ph.D. Thesis, Dept. of Mechanical Engineering. Univ. of Michigan, Ann Arbor, MI, 2002.

[28] Lophaven, S. N., Nielsen, H. B., and Søndergaard, J., "A Matlab Kriging Toolbox, Version 2.0," Informatics and Mathematical Modeling, Technical Univ. of Denmark, TR IMM-TR-2002-12, Lyngby, Denmark, 2002.

[29] iSIGHT, Software Package, Ver. 9.0, Engenious Software, Inc., Cary, NC, 2004

[30] Alexandrov, N., Dennis, J. E., Jr., Lewis, R. M., and Torczon, V., “A Trust Region Framework for Managing the use of Approximate Models in Optimization," Structural Optimization, Vol. 15, No. 1, 1998, pp. 16-23. doi:10.1007/BF01197433

[31] Jones, D. R., Schonlau, M., and Welch, W. J., "Efficient Global Optimization of Expensive Black-Box Functions," Journal of Global Optimization, Vol. 13, No. 4, Dec. 1998, pp. 455-492. doi:10.1023/A:1008306431147

[32] Knill, D. L., Giunta, A. A., Baker, C. A., Grossman, B., Mason, W. H., Haftka, R. T., and Watson, L. T., "Response Surface Models Combining Linear and Euler Aerodynamics for Supersonic Transport Design," Journal of Aircraft, Vol. 36, No. 1, 1999, pp. 75-86. doi: $\underline{10.2514 / 2.2415}$

N. Alexandrov Associate Editor 